\title{
Calibration of robotic manipulator systems for cone beam tomography imaging
}

\author{
Thomas Blumensath, Neil O'Brien, Charles E. Wood
}

\begin{abstract}
Iterative reconstruction of tomographic data relies on the precise knowledge of the geometric properties of the scan system. Common tomography systems such as rotational tomography, C-arm systems, helical scanners or tomosynthesis scanners generally use motions described by few rotational or linear motion axis. We are interested in applications in nondestructive testing, where objects might have large aspect rations and complex shapes. For these problems, more complex scan trajectories are required which can be achieved with robotic manipulator systems that have several linear or rotational degrees of freedom.

For the geometric calibration of our system, instead of using an approach that scans a calibrated phantom with markers at known relative position, we propose an approach that uses one (or several) markers with unknown relative positions. The fiducial marker is then moved by a known amount along one degree of freedom, thus tracing out a "virtual" phantom. Using the assumed spacial locations of the markers together with the locations of the markers on the imaging plane, we use a nonlinear optimisation method to estimate the orientation of the linear and rotational manipulator axis, the detector and source location and the detector orientation.
\end{abstract}

Index Terms-Laminography, geometric calibration, X-ray tomography

\section{INTRODUCTION}

$\mathrm{X}$-ray tomographic imaging technology is a valuable tool in medical diagnosis, scientific investigations, non-destructive testing and dimensional metrology. Traditionally, X-ray tomography typically relies on circular X-ray scanning trajectories where the X-ray source and detector perform a circular motion around the object under investigation. In helical scanning, a second, linear, motion is added. In many medical tomosynthesis systems and most semiconductor inspection laminography systems, the X-ray source and/or the sample perform rotational or linear motions, but now, the axis of rotation is no longer perpendicular to the central X-ray beam.

With these scan geometries, filtered back-projection type algorithms, such as the FDK algorithm [1] are often used as they are fast and work well if enough projections are available. For high quality image reconstruction, the rotation axis location and orientation needs to be known with high precision so that these imaging systems are regularly calibrated. Additionally, software corrections can often be performed after data has been acquired. For example, in traditional Computed

T. Blumensath and C.E. Wood are with the ISVR Signal Processing and Control Group, University of Southampton, SO17 1BJ, UK, Tel.: +44 (0) 23 80593224 ,e-mail: thomas.blumensath@soton.ac.uk, Neil O'Brien is with the $\mu$-VIS X-ray Imaging Centre, University of Southampton, UK

This research was supported by Innovate UK, the UK's innovation agency and the Aerospace Technology Institute (ATI), through grant 101804 (ProjecCAN) and EPSRC grant EP/R002495/1.

Thanks goes to the ProjectCAN consortium
Tomography (CT), the nominal position of the rotation axis can be shifted artificially in relation to the detector plane to provide sharper reconstructed volumetric images and some software packages use automated calibration routines that estimate the spatial orientation of the rotation axis.

The use of these X-ray tomography systems for nondestructive testing is limited by the shape of components that are inspected. Whilst traditional, circular scan trajectories work well for approximately cylindrical objects or small flat components such as circuit boards, some fossils or small flat panels, more complex objects require more flexible scan trajectories with more degrees of freedom [2], especially for high spatial resolution imaging with cone-beam systems, where magnification depends on the ratio between the source to detector distance and the source to object distance. High magnification thus requires the source to be close to the object surface, which is not achievable for many objects with complex shapes when using few rotational degrees of freedom.

To investigate the use of micro-focus X-ray tomography systems with complex scan trajectories, we have recently implemented two sample manipulator systems [3]. The first system uses a high precision hexapod and is shown in situ in figure 1. A hexapod consists of two plates connected by six linear actuators allowing the top plate to move, tilt and rotate relative to the bottom plate. To increase possible motions, the Hexapod in our system is mounted on a linear stage and an additional rotation stage was mounted on the top plate of the hexapod. The second system used a six axis robot arm manipulator as commonly used in the automotive industry.

To reconstruct volumetric images from data collected with these systems, iterative reconstruction methods are used. These require knowledge of the system and scan geometry, which is used to define the tomographic forward model. Whilst it is well known that these models might not be invertible or might be ill-conditioned unless the scan trajectories satisfy certain constraints [4], we could show that even highly undersampled data can lead to useful reconstructions where full CT is impossible [3]. However, any uncertainty in the specification of the system geometry will lead to a reduction in spatial image resolution. Methods to measure these parameters are thus crucial.

\section{A. Contribution}

This paper looks at the problem of geometric calibration of a robotic tomographic sample manipulator. We propose an approach where calibration scans are performed to provide geometric information and to link this to the positional encoder data available from the manipulator's position encoders. By 


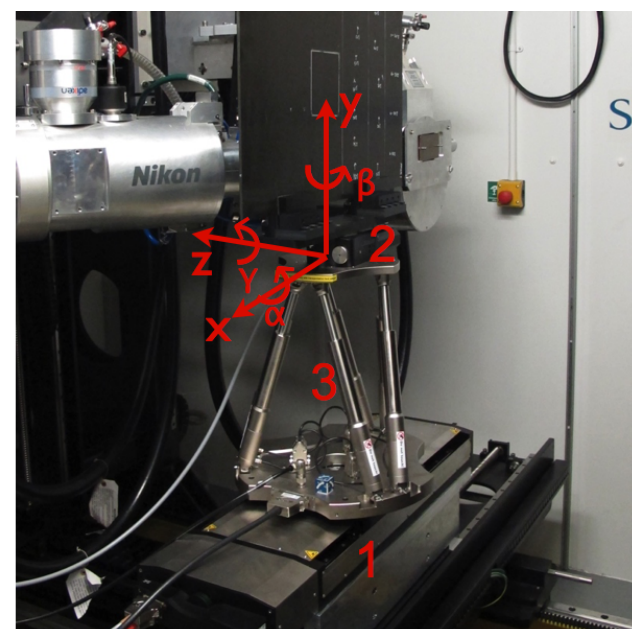

Fig. 1: Hexapod based manipulator in our custom build Xray room with a $225 \mathrm{kV}$ micro-focus $\mathrm{X}$-ray source (left) and a $400 \mathrm{~mm}$ by $400 \mathrm{~mm}$ flat panel detector (out of shot to right). A linear stage (1) holds a six axis hexapod (3) on which an additional rotation stage is mounted (2). A $600 \mathrm{~mm}$ by $600 \mathrm{~mm}$ carbon fibre panel is shown mounted on our manipulator ready for laminographic imaging of a $40 \mathrm{~mm}$ by $40 \mathrm{~mm}$ region of interest. The system allows the linear translation of the sample ( $\mathrm{x}, \mathrm{y}$ and $\mathrm{z}$ direction). The hexapod also allows rotation of the top platform around the three orthogonal axis $(\alpha, \beta$ and $\gamma)$ ). The rotation stage offers an additional axis of rotation to extend the limited rotation range available by the hexapod. Note, the hexapod rotation axis $\beta$ might not be aligned with the rotation stage rotation axis and the hexapod linear axis $\mathrm{x}$ might not be aligned with the axis of the linear stage (1). In our scans, source, detector and hexapod system (who sit on their own linear axes, are moved so that a region of interest on the carbon fibre panel is centred in the X-ray projection image. The detector and the hexapod assembly are moved towards or away from the X-ray source to set the require magnification and clearance. During scanning, source and detector remain fixed whilst the sample is moved.

detecting and tracking features in the projection images during the calibration scan, we describe optimisation algorithms that use the manipulator's reported position to estimate source and detector location, detector orientation and the position and orientation of linear and rotational axes of the manipulator system.

Calibration in traditional, rotation based, X-ray tomography systems has been studied before. Purely data driven approaches try to find the rotation axis alignment based on projections that are offset by 180 degree [5], or optimise quality measures such as entropy to improve the spatial resolution of the reconstructed image [6], thus estimating system parameters in the process. An alternative is the work in [7] where a geometrical model of the object is used for calibration. Another set of approaches uses calibration phantoms or other measurement devices. For example, [8] and [9] use fixed test objects for calibration, whilst atomic force microscopy has been used in [10].
Geometric calibration for tomosynthesis systems has been studied in [11], [12] and [13]. Calibration of these systems is done using a phantom with several markers. The relative position of the markers in the phantom is assumed known. A full scan is then performed of the phantom and for each marker, the marker centre is estimated in each projection image. For each projection, a linear transform is then estimated that maps the centre of each marker in 3D space to to the centre of the marker's projection centre [11]. This provides sufficient geometric information to either correct each projection image before a filtered backprojection reconstruction is computed or to compute an accurate system matrix for each projection to use in algebraic reconstruction. Different types of markers are used commonly in the construction of calibration phantoms. Tungsten [12], steel [14] or ruby [15] spheres are common and so are hole plates [16]. A detailed analysis of the influence of different sources of errors on the accuracy of this approach to geometric calibration can be found in [17] and [18].

The calibration approach of [11] has also been used in robotised inspection [19]. Calibration based on the method in [11], however, relies on the availability of a dedicated phantom with markers that are either placed with high precision or whose location can be measured accurately. We propose an approach that does not rely on such a phantom. Instead, we assume that we have a manipulator that is either manufactured to the required precision or whose movement accuracy has been measured. Our work builds on the work in [8] and [9]. As in [8] and [11], we use calibration scans. However, in contrast to the approach in [11], instead of estimating the geometry for each projection, we only estimate the location and orientation of the linear (translational) and rotational axes along and around which the manipulator (and or the source and the detector) move.

Our calibration scans will use one or several small $(2 \mathrm{~mm})$ chrome steel spheres. Spheres can either be mounted on an $\mathrm{X}$-ray transparent object and attached to the manipulator or they can be attached directly to the object that is to be scanned (either directly using tape or mounted on a acrylic block which in turn is taped to the object). To estimate the orientation and location of linear axis of motion, we use the sample manipulator to move the sphere to different positions within the X-ray cone-beam. This is equivalent to the use of a test phantom constructed of several spheres placed at fixed and known distances and angles. Uncertainty in the accuracy of sphere location now depends on the accuracy of the sample manipulator system. This uncertainty could be assessed using co-ordinate measurement machines, though in this work we rely on the encoder accuracy in the manipulator and thus assume that the reported locations are the actually achieved locations. An evaluation of biases, non-linearities and variances in the sample manipulator's accuracy remains to be undertaken in the future.

The advantage of our approach is that we do not need a calibrated phantom, however, we do assume the system's motion is accurately modelled with several linear or rotational degrees of freedom the movement along which is accurately measured by the manipulator itself. Deviations from these assumptions cannot be corrected for with our approach. 
As in [9] and [11], we define the geometry of our system with all its degrees of freedom and use an optimisation method to optimise the geometric parameters based on an estimate of the centres of the projected spheres. The difference to [9] is that our geometry is less constrained than the traditional cone-beam tomography geometry with a single rotation axis. In contrast to the approach in [11], our aim is the estimation of the axes of motion, rather than the linear projection matrix estimated in [11]. Due to the non-linear relationship between the movements along and around these axes and the location of marker centres in the projection image, we do not have a linear system that can be solved with a direct method. Instead, we have a non-linear optimisation problem that we will solve with co-ordinate descent.

\section{B. Notation}

We will use bold face roman characters to specify vectors in three dimensional Euclidean space. In particular,

- $\mathbf{S}$ - is the source location

- $\mathbf{D}_{0}$ - is the detector location (specified as either the detector centre or as one of the corner pixels)

- $\mathbf{u}, \mathbf{v}$ and $\mathbf{n}$ are orthogonal vectors that define the detector orientation, $\mathbf{u}$ points along detector rows and $\mathbf{v}$ points along detector columns

- $\mathbf{x}, \mathbf{y}$ and $\mathbf{z}$ are orthonormal co-ordinate axis that define the main co-ordinate system we will be using.

- $\mathbf{P}$ will be a generic point in space, such as the location of the calibration sphere.

- $\mathbf{d}(\mathbf{P})$ will be a two dimensional vector that defines the location of the projection of point $\mathbf{P}$ in terms of the two row and column detector co-ordinate vectors. We will write $d_{1}$ as the component of $\mathbf{d}(\mathbf{P})$ in the direction $\mathbf{u}$ and $d_{2}$ as the component of $\mathbf{d}(\mathbf{P})$ in the direction $\mathbf{v}$.

All vectors will be in relation to a co-ordinate system with zero point at one of the sphere locations in one of the calibration scan locations. The directions of the coordinate axis are then the directions of the linear sample manipulator movement axes, which are assumed to be orthogonal.

\section{SyStem SETUP}

The geometry of an X-ray cone-beam projection system can be defined by the source location $\mathbf{S}$, the location of the detector $\mathbf{D}_{0}$ and by a local co-ordinate system on the detector defined by orthogonal vectors $\mathbf{u}$ and $\mathbf{v}$, so that each point on the detector can be written as $\mathbf{D}_{P}=\mathbf{D}_{0}+d_{1} \mathbf{u}+d_{2} \mathbf{v}$. Note that $\mathbf{u}$ and $\mathbf{v}$ also define the orthogonal vector $\mathbf{n}=\mathbf{u} \times \mathbf{v}$. In general, source and detector locations can change from projection to projection, though for simplicity, we assume that the source and detector location and orientation remain fixed during the scan $^{1}$.

The manipulator is assumed to provide linear motions along three orthogonal axis $\mathbf{x}, \mathbf{y}$ and $\mathbf{z}$ as well as rotational motion around a rotation axis $\mathbf{r}$. We restrict the derivation to a single

\footnotetext{
${ }^{1}$ To extend our approach to allow movement of source and detector, additional calibration scans that link the source and detector movement axes to the system co-ordinates can be introduced.
}

rotation axis, though the extension to several axes can follow a similar approach to the one we use here. We assume the three linear axes $\mathbf{x}, \mathbf{y}$ and $\mathbf{z}$ form the basis of our global co-ordinate system, that is, we express all other vectors as combinations of $\mathbf{x}, \mathbf{y}$ and $\mathbf{z}$.

\section{CALIBRATION SCANS}

In order to perform geometric calibration, we either need a calibrated test object with several features distributed in space or we use an object with a single feature that is moved to different spatial locations using the manipulator. We follow the second approach which requires some form of calibration of the manipulator itself. Whilst we have done some initial calibration experiments with one of our manipulators, we assume that the accuracy of the manipulators is high enough and that the positional readouts from the internal positional encoders is accurate to the required precision.

As test object, we used either ruby spheres or $2 \mathrm{~mm}$ grade 10 hardened 52100 Chrome Steel ball bearings that were either taped to the test object or glued onto some other low attenuating holder which in turn was taped to he test object. Whilst the cone-beam projections of spheres are not exactly circular [20], we make this assumption.

Our experiments are performed in a purpose build Nikon metrology X-ray room. We use a Nikon $225 \mathrm{kV}$ micro focus X-ray source, a Perkin Elmer XRD 1621 xN3 ES detector with CsI scintillator (16 bit, $0.2 \mathrm{~mm}$ pixel size and $2048 \mathrm{x}$ 2048 pixel image size). Tube voltage was set to between 80 and $90 \mathrm{kV}$ with power adjusted to between $13 \mathrm{~W}$ and $52 \mathrm{~W}$, depending on the object in the X-ray beam.

\section{A. Calibration of linear axis}

We use a single sphere to estimate source location $\mathbf{S}$, detector location $\mathbf{D}_{0}$ and detector orientation (roll, yaw and pitch) specified by the orthogonal vectors $\mathbf{u}$ and $\mathbf{v}$. This is done relative to the three linear axes of the manipulator $\mathbf{x}$, $\mathbf{y}$ and $\mathbf{z}$ which form the orthogonal basis of our co-ordinate system. A calibration scan is used where projection images are taken at different spatial positions with different values for $\mathbf{x}$, $\mathbf{y}$ and $\mathbf{z}$. We use 18 projections, taken with the manipulator performing linear motions to points $x \mathbf{x}+y \mathbf{y}+z \mathbf{z}$, where $x \in\{-d x, 0,+d x\}, y \in\{-d y, 0,+d y\}$ and $z \in\{-d z, 0\}$. The values for $d x, d y$ and $d z$ depend on the magnification used in the scan and are chosen so that all points are projected onto the detector. We did not use $z=+d z$ to avoid collision with the X-ray source.

In our experiments, we scanned a range of composite panels of varying dimensions (with thicknesses between $5 \mathrm{~mm}$ and $10 \mathrm{~mm}$ and hight and width between $20 \mathrm{~mm}$ by $20 \mathrm{~mm}$ to $600 \mathrm{~mm}$ by $600 \mathrm{~mm}$. Panels were made predominately of carbon fibre reinforced polymer, but some contained additional copper mesh layers and different aerospace paint systems. For the calibration scan, the sphere was attached the composite panel (the sample). 


\section{B. Calibration of rotation axis}

To calibrate the rotation axes, we use the same sphere attached to the same location on the sample as in the calibration of the linear axis. We also use two additional spheres mounted to the manipulator in different positions. We then rotate the axis through a range of rotational offsets (including a rotation of 0 ). The position of the sphere used for both, linear and rotational calibration should be in the same position when the rotation is 0 and the linear shift is 0 . This is important to allow us to link the position of the rotation axis to the co-ordinate system $\mathbf{x}, \mathbf{y}$ and $\mathbf{z}$. Both calibration scans are thus performed one after the other with at least one sphere staying in place.

\section{Analysing the projection data}

We estimate the centres of the balls by manually identifying the different features in the image. This is done using a graphic user interface in which the user clicks on the sphere in each image. A mask is generated around this pixel that is large enough to contain the entire sphere. Once the different features are identified in each image, a neighbourhood of each feature is selected using the masks. This region is then filtered using a gaussian filter with a standard deviation of five pixels before canny edge detection detects the outline of the spheres. After outlier detection, the edge points are fitted with a circle whose centre is used as the centre of the projected sphere.

The results are shown in figure 2, where we show six different projection images after linear (top) and rotational (bottom) displacement of the sample. Overlaid are all estimated sphere centres for the linear (top) and rotational (bottom) motions ${ }^{2}$. For the linear motion, we have used a single sphere to trace a 3 by 3 grid of locations at two magnifications. For the rotation, we have use seven projections at different rotation angles and estimated the centres of three spheres whenever these were projected onto the detector (On occasion, a sphere might be rotated fully or partially outside the cone beam so that no data is available for this sphere for this projection). Centres for different spheres are shown in different colours. In the example shown in figure 2, the spheres were attached to the object that was to be scanned subsequently. The object here is a $300 \mathrm{~mm}$ by $300 \mathrm{~mm}$ by $10 \mathrm{~mm}$ carbon fibre panel with a copper mesh layer as used in composite aircraft manufacture. One sphere was taped directly to the panel (the lower sphere in all images) whilst the other two spheres were attached with epoxy glue to a perspex block into which two shallow conical holes were machined to better secure the spheres. The epoxy block was then taped to the sample. The shadow of the epoxy block is visible in figure 2 as a darker square region.

\section{GeOMETRIC CALIBRATion}

To estimate the system's geometric parameters, we model the centre of each sphere as a point in 3D space, which we parameterise as a function of the unknown system parameters and the linear shifts or rotation angles. We then model the

\footnotetext{
${ }^{2}$ Note that when plotting the crosses that identify sphere centres, the crosses are not exactly centred on the spheres. This is an artefact of plotting the figures and does not reflect the actual estimates of the sphere centres.
}
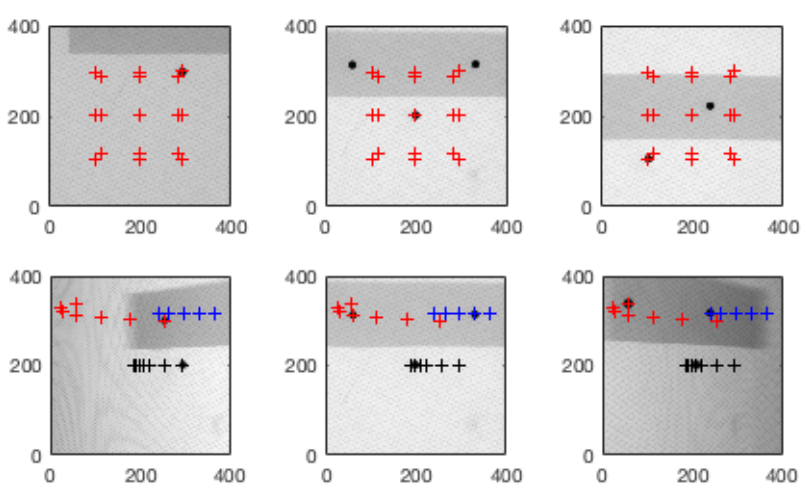

Fig. 2: Flat field corrected and log transformed x-ray intensity images at three different linear off-set locations (top) and three rotations (bottom) for a region of interest view of a carbon fibre panel with a coper mesh layer. The panel extends across the entire image in all views. Three chrome steel ball bearings (dark circles) are attached, two mounted on a perspex block (darker grey area in the images). X-ray source power and voltage were adjusted to give a good contrast between the ball bearings and the panel. For the linear off-set calibration, we only use the lower sphere. The projection of the sphere and its identified location $(+)$ are shown (from left to right) in the top right, central and bottom left locations. The other (+) in the top row indicate the estimated sphere locations for the other off-sets. In the bottom row, we show projections with three rotational off-sets. We use all three spheres for calibration and the estimated locations of the sphere centres are shown with different colours labelling different spheres. Axis labels are in $\mathrm{mm}$. The copper mesh pattern produces aliasing artefacts in the low resolution images displayed in the pdf version of this manuscript, these are not visible in the original images.

projection of these points onto the detector plane. We optimise the unknown system parameters to minimise the distance between the predicted locations of the points on the plane and the actually observed centre positions of the projected spheres.

\section{A. Cone-beam projection}

Let us start by modelling the projection of a point in 3D space onto the detector. For any point $\mathbf{P}$ that lies between the source and the detector, the cone-beam projection of $\mathbf{P}$ onto the detector can then be written in two ways:

$$
\mathbf{D}_{0}+d_{1}(\mathbf{P}) \mathbf{u}+d_{2}(\mathbf{P}) \mathbf{v}
$$

and

$$
\mathbf{S}+\alpha(\mathbf{P}-\mathbf{S}),
$$

where $\alpha$ is a positive scalar. This is shown in figure 3. Let us also define the point $\mathbf{D}_{S}$, which is the orthogonal projection of the source location onto the detector. To derive an expression for $\alpha$, we can then consider the two triangles $\overline{\mathbf{D}_{0} \mathbf{S D}_{S}}$ and $\overline{\mathbf{D}_{P} \mathbf{S D}_{S}}$, which share the side $\overline{\mathbf{S D}_{S}}$.

Note that the line $\overline{\mathbf{S D}_{S}}$ is parallel to the normal of the detector plane, which is defined as $\mathbf{n}=\mathbf{u} \times \mathbf{v}$. Let us assume that $\mathbf{u}$ and $\mathbf{v}$ are orthonormal. This implies that $\mathbf{n}$ is of unit 


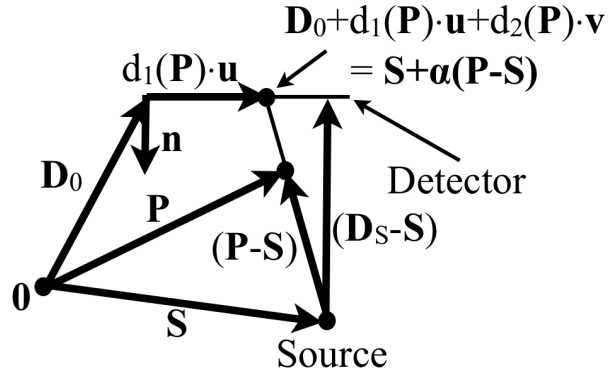

Fig. 3: 2D view along the direction $\mathbf{v}$ of the geometry of the cone-beam projection setting.

length as well. Let us also assume that $\mathbf{n}$ points from the detector towards the source, that is, $\left\langle\mathbf{D}_{S}-\mathbf{S}, \mathbf{n}\right\rangle<0$, where $\langle\cdot, \cdot\rangle$ is the inner product. The length of the vector $\overline{\mathbf{S D}_{S}}$ is thus $-\left\langle\mathbf{D}_{0}-\mathbf{S}, \mathbf{n}\right\rangle$. Similarly, the component of $\mathbf{P}-\mathbf{S}$ in direction $\mathbf{n}$ is $|\langle\mathbf{P}-\mathbf{S}, \mathbf{n}\rangle|$, where the negative sign comes from the assumption that $\mathbf{P}$ lies between the source and the detector. If we instead assume that $\mathbf{n}$ points away from the source, then the signs are in the opposite direction. In either case, the ratio $\left\langle\mathbf{D}_{0}-\mathbf{S}, \mathbf{n}\right\rangle /\langle\mathbf{P}-\mathbf{S}, \mathbf{n}\rangle$ is always positive whenever the points $\mathbf{P}$ lie between the source and the detector.

As $\overline{\mathbf{D}_{P} \mathbf{S D}_{S}}$ and $\overline{\mathbf{S P S}+\mathbf{n}\langle\mathbf{P}-\mathbf{S}, \mathbf{n}\rangle}$ are similar, we have the relationship:

$$
\alpha \frac{\|\mathbf{P}-\mathbf{S}\|}{\left\|\mathbf{D}_{S}-\mathbf{S}\right\|}=\frac{\|\mathbf{P}-\mathbf{S}\|}{\|\mathbf{n}\langle\mathbf{P}-\mathbf{S}, \mathbf{n}\rangle\|}=\frac{\|\mathbf{P}-\mathbf{S}\|}{-\langle\mathbf{P}-\mathbf{S}, \mathbf{n}\rangle}
$$

Which, using $\left\|\mathbf{D}_{S}-\mathbf{S}\right\|=-\left\langle\mathbf{D}_{0}-\mathbf{S}, \mathbf{n}\right\rangle$ means that

$$
\alpha=\frac{\left\langle\mathbf{D}_{0}-\mathbf{S}, \mathbf{n}\right\rangle}{\langle\mathbf{P}-\mathbf{S}, \mathbf{n}\rangle} \text {. }
$$

We thus have the following equation that links the detector co-ordinate location of a projected point to the location of this point:

$$
d_{1}(\mathbf{P}) \mathbf{u}+d_{2}(\mathbf{P}) \mathbf{v}=\left(\mathbf{S}-\mathbf{D}_{0}\right)+\frac{\left\langle\mathbf{D}_{0}-\mathbf{S}, \mathbf{n}\right\rangle}{\langle\mathbf{P}-\mathbf{S}, \mathbf{n}\rangle}(\mathbf{P}-\mathbf{S}) .
$$

Taking inner products with $\mathbf{u}$ and $\mathbf{v}$ and using the fact that $\langle\mathbf{u}, \mathbf{v}\rangle=0$ and $\langle\mathbf{u}, \mathbf{n}\rangle=0$, we thus get the following equations:

$$
\mathbf{d}(\mathbf{P})=\left[\begin{array}{l}
d_{1}(\mathbf{P}) \\
d_{2}(\mathbf{P})
\end{array}\right]=\left[\begin{array}{l}
\left\langle\mathbf{S}-\mathbf{D}_{0}, \mathbf{u}\right\rangle+\frac{\left\langle\mathbf{D}_{0}-\mathbf{S}, \mathbf{n}\right\rangle}{\langle\mathbf{P}-\mathbf{S}, \mathbf{n}\rangle}\langle\mathbf{P}-\mathbf{S}, \mathbf{u}\rangle \\
\left\langle\mathbf{S}-\mathbf{D}_{0}, \mathbf{v}\right\rangle+\frac{\left\langle\mathbf{D}_{0}-\mathbf{S}, \mathbf{n}\right\rangle}{\langle\mathbf{P}-\mathbf{S}, \mathbf{n}\rangle}\langle\mathbf{P}-\mathbf{S}, \mathbf{v}\rangle
\end{array}\right]
$$

This links the location of any point $\mathbf{P}$ to the location of the projection of this point in terms of the detector co-ordinates $\mathbf{u}$ and $\mathbf{v}$. This mapping is a function of the unknown geometric parameters $\mathbf{S}, \mathbf{D}_{0}, \mathbf{u}$ and $\mathbf{v}$.

\section{B. Mapping linear and rotational motion to points in $3 D$ space}

We assumed that the linear motion defines a linear coordinate system with three orthonormal axes in 3D. We thus assumed we know the locations of the points $\mathbf{P}$ for the different linear motions.

For the rotational motion, we know that one of the points $\mathbf{P}$ is at the centre of the co-ordinate system. However we do not know the spatial position of the other points, all we know is that they are rotated versions of points in 3D space (some are rotated versions of the zero point). Importantly, the rotation is around an unknown axis of rotation which needs to be estimated.

For sphere $i$, let $\mathbf{P}_{i}^{k}$ be the actual location of the centre of the sphere in 3D space after a rotation by some angle $\theta_{k}$. Each sphere is assumed to be rotated around the same rotational axis. We use the following way to describe the location of points $\mathbf{P}_{i}^{k}$ in terms of this axis

$$
\mathbf{P}_{i}^{k}=\mathbf{p}_{0}+\alpha_{i} \mathbf{r}+a_{i} \mathbf{q}_{i}^{k},
$$

where $\mathbf{r}$ is the axis of rotation, $\mathbf{p}_{0}$ specifies the location of this axis in relation to our co-ordinate system and vectors $\mathbf{q}_{i}^{k}\left(\theta_{i}^{k}\right)$ are unit vectors that are orthogonal to the rotation axis which are rotated around this axis by $\theta^{k}$ relative to some initial vector $\mathbf{q}_{i}^{k}(0)$. As this model is over-specified, we assumed that $\mathbf{r}$ and $\mathbf{q}_{i}^{k}\left(\theta_{i}^{k}\right)$ are unit vectors. Furthermore, we fix one of the co-ordinates of $\mathbf{p}_{0}$. We chose the direction which is roughly parallel to the expected axis of rotation. The parameter $a_{i}$ thus specifies the distance of the sphere from the axis of rotation. Similarly, the parameters $\alpha_{i}$ specify the height of the plane of rotation along $\mathbf{r}$ above point $\mathbf{p}_{0}$.

Let $\mathbf{I}=[1,0,0]^{T}$. To define the vectors $\mathbf{q}_{i}^{k}$, we define

$$
\mathbf{q}_{o}(\mathbf{r})=\frac{\mathbf{I}-\mathbf{r} /\|\mathbf{r}\|^{2} \mathbf{I}^{T} \mathbf{r}}{\|\mathbf{I}-\mathbf{r} /\| \mathbf{r}\left\|^{2} \mathbf{I}^{T} \mathbf{r}\right\|}
$$

which is a unit norm vector orthogonal to $\mathbf{r}$ (We assumed that the rotation axis is not parallel to the $\mathrm{x}$ axis of our co-ordinate system). Let $R_{\theta}(\mathbf{P})$ be the affine transformation that describes the rotation of a point $\mathbf{P}$ around the axis defined by $\mathbf{p}_{0}$ and $\mathbf{r}$. The vectors $\mathbf{q}_{i}^{k}$ are then

$$
\mathbf{q}_{i}^{k}=R_{\theta_{i}^{0}+\theta^{k}}\left(\mathbf{q}_{o}\right)
$$

where $\theta^{k}$ is the rotation angle used when taking the $k^{t h}$ projection and where $\theta_{0}^{i}$ is an initial rotation that depends on the location of the sphere relative to the rotation axis.

With this model, we thus have to estimate the following parameters: $\mathbf{p}_{0}, \mathbf{r}, \alpha_{i}, a_{i}$ and $\theta_{i}^{0}$. The first two vectors specify the location and orientation of the rotation axis, whilst the other parameters define the location of the spheres relative to this axis.

\section{ESTIMATION OF PARAMETERS FROM LINEAR MOTIONS}

In the calibration experiment a sphere is moved to different points along three orthogonal axes. We assume that we know the distances between the sphere centres after each motion. We can then define a lab co-ordinate system, that is, we have a co-ordinate system in which the centre locations are known, that is, we know $\mathbf{P}_{1}, \mathbf{P}_{2}, \cdots, \mathbf{P}_{N}$. Assume all spheres have been projected onto the detector to points $\mathbf{d}\left(\mathbf{P}_{1}\right), \mathbf{d}\left(\mathbf{P}_{2}\right), \cdots, \mathbf{d}\left(\mathbf{P}_{N}\right)$ which have been estimated using, for example, our circle fitting procedures. Assuming the detector rows and columns are orthogonal, we define $\mathbf{D}_{0}$ to be the bottom left (as seen from the source) pixel location in the lab co-ordinate system. We define $\mathbf{u}$ to point along the pixel rows and $\mathbf{v}$ along pixel columns. The estimated centres of the 
sphere projections are given in detector co-ordinates, that is, for sphere $i$, we have a vector $\mathbf{d}_{i}$.

To estimate the geometry of the cone-beam projection, we define the following cost function:

$$
\frac{1}{N} \sum_{i}\left\langle\mathbf{d}_{i}-\mathbf{d}\left(\mathbf{P}_{i}\right), \mathbf{d}_{i}-\mathbf{d}\left(\mathbf{P}_{i}\right)\right\rangle .
$$

We would like to minimise this cost with respect to the vectors $\mathbf{S}, \mathbf{D}_{0}, \mathbf{u}$ and $\mathbf{v}$ under the constraints that

$$
\begin{aligned}
& \langle\mathbf{u}, \mathbf{u}\rangle=1, \\
& \langle\mathbf{u}, \mathbf{v}\rangle=0, \\
& \mathbf{n}=\mathbf{u} \times \mathbf{v}
\end{aligned}
$$

and

$$
\frac{\left\langle\mathbf{D}_{0}-\mathbf{S}, \mathbf{n}\right\rangle}{\langle\mathbf{P}-\mathbf{S}, \mathbf{n}\rangle}>0 .
$$

Different approaches to the optimisation of this cost function are possible. We could use a gradient descend method, however, exploratory experiments have shown that a carefully designed co-ordinate descend method with line search procedure was faster and this is the approach we present here.

We initialise all unknown geometric parameters with estimates taken from our scanner's geometric setup. We have set up our system so that two of the axis of the manipulator run roughly parallel to the two axes of the detector. We also have mounted the sphere so that it is projected close to the centre of the detector. We thus initialise

- $\mathbf{u}$ is set to the $\mathrm{x}$-direction of our manipulator axis

- $\mathbf{v}$ is set to the y-direction of our manipulator axis.

- the position of the source location is set to zero in the $x$ and y direction

- the detector position $\overline{\mathbf{D}_{0}}$ is set to zero in the $\mathrm{x}$ and $\mathrm{y}$ direction

- the source to object and source to detector distances are set to arbitrary values which in turn define the source and detector $\mathrm{z}$ coordinates.

We know that point $\mathbf{P}_{0}=[0,0,0]^{T}$ projects onto the detector at point $\mathbf{d}_{0}$. We thus constrained our optimisation so that this is always satisfied. To do this, we start optimisation by moving the detector location $\mathbf{D}_{0}$ so that the point $[0,0,0]^{T}$ projects onto $\mathbf{d}_{0}$. To simplify notation, we re-parameterise the detector co-ordinate system by defining the zero point of the detector co-ordinate system to be the point on the detector onto which the first sphere is projected. Let $\overline{\mathbf{D}_{0}}$ be that point, that is $\overline{\mathbf{D}_{0}}=\mathbf{D}_{0}+\mathbf{d}_{0}[1] \mathbf{u}+\mathbf{d}_{0}[2] \mathbf{v}$. Let $\mathbf{d}_{i}$ be the adjusted coordinates of the measured projections such that

$$
\mathbf{D}_{0}+\mathbf{d}_{i}[1] \mathbf{u}+\mathbf{d}_{i}[2] \mathbf{v}=\overline{\mathbf{D}_{0}}+\overline{\mathbf{d}}_{i}[1] \mathbf{u}+\overline{\mathbf{d}}_{i}[2] \mathbf{v}
$$

With this parameterisation, we can then optimise system properties one at a time whilst keeping the projection of $\mathbf{P}_{0}$ fixed on point $\overline{\mathbf{D}_{0}}$. $\mathbf{S}$ and $\overline{\mathbf{D}_{0}}$ are defined through the vectors $\mathbf{S}$ and $\overline{\mathbf{D}_{0}}-\mathbf{S}$ which are parallel as the points $\mathbf{S}, \overline{\mathbf{D}_{0}}$ and $[0,0,0]^{T}$ lie on a line by definition. If we move $\mathbf{S}$ along this line closer to $\overline{\mathbf{D}_{0}}$, we in effect change the magnification of the projections. If we move both $\mathbf{S}$ and $\overline{\mathbf{D}_{0}}$ so that the ratio

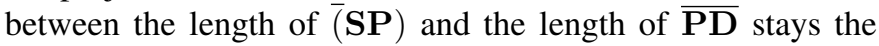

same, then the magnification stays the same but we change the effective cone angle used in the projection. We can also rotate $\mathbf{S}$ and $\overline{\mathbf{D}_{0}}$ around the co-ordinate system centre (this gives us two parameters to optimise) and we can rotate $\mathbf{u}$ and $\mathbf{v}$ around point $\overline{\mathbf{D}_{0}}$ (giving two more parameters).

We then iteratively use bracketing line search procedures [21] to optimise

1) Magnification

2) Cone angle

3) Rotation of $\mathbf{S}, \overline{\mathbf{D}_{0}}$ and $\mathbf{v}$ around an axis parallel to $\mathbf{u}$ going through the coordinate centre.

4) Rotation of $\mathbf{S}, \overline{\mathbf{D}_{0}}$ and $\mathbf{u}$ around an axis parallel to $\mathbf{v}$ going through the coordinate centre.

5) Rotation of $\mathbf{u}$ and $\mathbf{v}$ around $\overline{\mathbf{D}_{0}}$ keeping $\mathbf{u}$ and $\mathbf{v}$ in the detector plane.

6) Rotation of $\mathbf{v}$ around $\overline{\mathbf{D}_{0}}$ and axis $\mathbf{u}$.

In the first iteration, we only optimise based on the projections for which the points $\mathbf{P}$ where in one plane, i.e. $\mathbf{P}_{i}=[\cdot, \cdot, 0]^{T}$ which provided a rough initial estimate of all parameters apart from the source to detector distance.

We then optimise with respect to all parameters, using bracketed line search optimise one randomly selected parameter at a time. This is in effect a co-ordinate descent method to optimise the non-linear cost function [21].

\section{OPTIMISATION OF ROTATION AXIS}

Once a co-ordinate system has been established together with the source and detector locations as well as the detector co-ordinate axis, the use of rotations in many tomographic trajectories requires the estimation of one or several rotation axis.

We again parameterise the problem so that $\mathbf{P}_{0}$ projects onto $\overline{\mathbf{D}_{0}}$ and get initial estimates of the system parameters by

- Setting the rotation axis is parallel to the y axis.

- Initialising orientation and location of the axis by optimising only the projections from the sphere that at $\theta_{0}^{0}$ is at point $\mathbf{P}_{0}$.

- Estimating $\alpha_{0}$ by projecting the initial estimate of the rotation axis onto the detector assuming $\mathbf{r}$ to be known.

- Projecting all points $\mathbf{d}\left(\mathbf{P}_{i}\right)$ on the projector onto this projection of the rotation axis. This provides an initial estimate of $\alpha_{0}$.

This is done for all spheres

We then use co-ordinate wise bracketing line search to estimate the rotation axis location and orientation.

During the optimisation, we fix the position of the rotation axis by using the fact that one sphere is at point $[0,0,0]$ at a rotation of 0 . This constraint defines the following equation

$$
\mathbf{0}=\mathbf{p}_{0}+\alpha_{1} \mathbf{r}+a_{1} \mathbf{q}_{i}^{0},
$$

which allows us to compute $a_{1}, \mathbf{p}_{0}[1]$ and $\mathbf{p}_{0}[3]$ for any $\alpha_{1}$ a $\theta_{0}$. Remember that $\theta_{0}$ defines the vector $\mathbf{q}_{i}^{0}$ in relation to r. In fact, we use the above equality every time we update $\theta_{0}$ and $\mathbf{q}_{i}^{0}$ to update the location of the rotation axis.

We then estimate the vector $\mathbf{q}_{0}$ for this sphere which is parameterised through two parameters, an initial rotation around $\mathbf{r}$ and its length $a_{0}$. We perform a two stage line search, 
for each proposed value of $a_{0}$, we run a bracketed line search to find the optimal initial rotation. The cost is always evaluated such that for any combination of length and initial rotation, the vector $\mathbf{p}_{0}$ is shifted so that point $\mathbf{P}_{0}=[0,0,0]^{T}$. Note that the initial rotation cost will be a circular cost function that is $2 \pi$ periodic. The initial bracket for the line search is thus always made up of two points that are $2 \pi$ apart plus a point between them that has a lower cost. This way we are able to explore all possible values of rotation. Also, sometimes, the line search for the length of the vector returns a negative value at which point we change the sign of this length together with the initial rotation angle to which we add $\pi$. Initially this optimisation is done looking only at the error between the locations of the projected sphere centres for this one sphere. Optimisation is stopped once this distance is at a minimum and does not change significantly any more.

After this initial optimisation, we have an estimate of $\mathbf{p}_{0}$ which we then use to initialise the position of the other spheres. The only difference is that we also do an additional bracketed line search for the values of $\alpha_{i}$. We first optimise the initial rotation of the vector and then optimise $\alpha_{i}$, both using bracketed line searches.

Fine tuning for these parameters is performed similar to the approach used for the linear axis using a randomised coordinate descend algorithm with bracketed line search, with the difference that now the dependence of the projected sphere locations on the rotation axis parameters follows a different form. Note that when estimating $a_{0}$ and $\theta_{0}$ we are automatically updating $\mathbf{p}_{0}$ as well. Only once the cost function has converged with sufficient precision do we add bracketed line searches that rotate the rotation axis $\mathbf{r}$ around $\mathbf{q}_{0}(\mathbf{r})$ and $\mathbf{p}_{0}(\mathbf{r})=\mathbf{r} \times \mathbf{q}_{0}(\mathbf{r})$.

\section{RESULTS}

To analyse the performance of the method, we performed several calibration scans using both manipulators. Several aspects of the method are of interest and we explore the convergence, how well the method works over a range of scans, how repeatable the measurements are and, finally, how much the calibration improves image quality. The main performance measure we are using will be in terms of the average error between the measured locations of the projections of the spheres and the estimated projections for the assumed geometry. Detector pixels were $0.2 \mathrm{~mm}$ wide and high and we express the error also in $\mathrm{mm}$. Thus, errors below $0.2 \mathrm{~mm}$ are smaller than the pixel size.

\section{A. Convergence}

1) Calibrating linear axis: To see how well the method performs and converges, we show the difference between measured and estimated centre locations on the detector in figure 4 . The first panel shows the difference after an initial estimation of the magnification and cone angle whilst the second panel shows the final result after 35 iterations. Figure 5 shows the speed with which the method converges. It only takes about 5 iterations to get a sub-pixel average error.

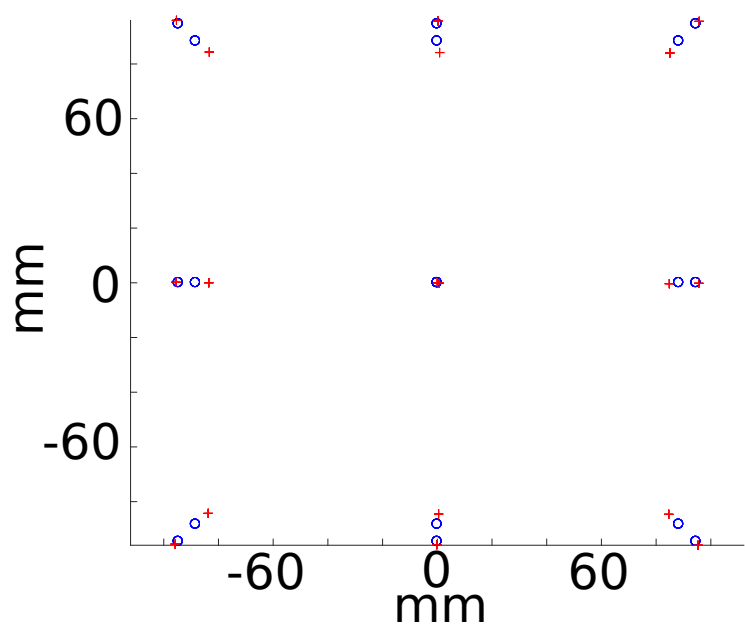

(a) After initial step

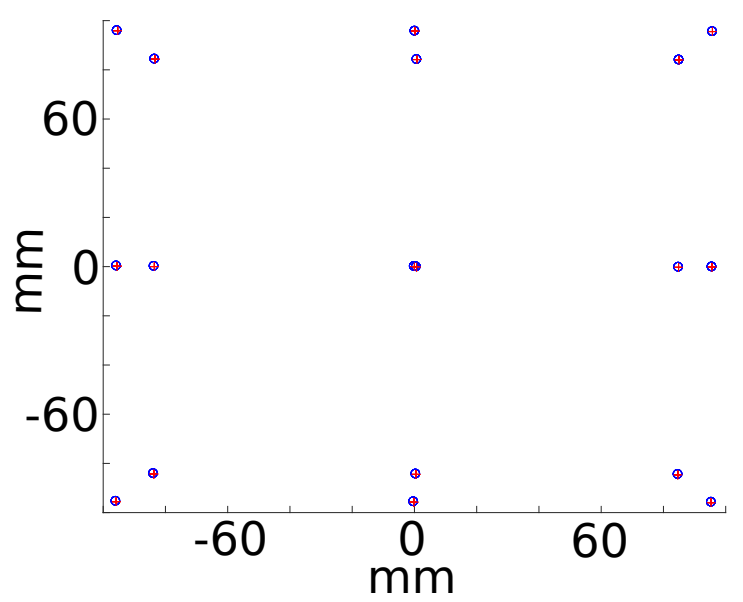

(b) After 35 iterations

Fig. 4: Measured sphere centres on detector (red +) and estimated centres for optimised geometry (blue circle). Average distance between observed and estimated centres is $0.092 \mathrm{~mm}$, which is half the width of a pixel.

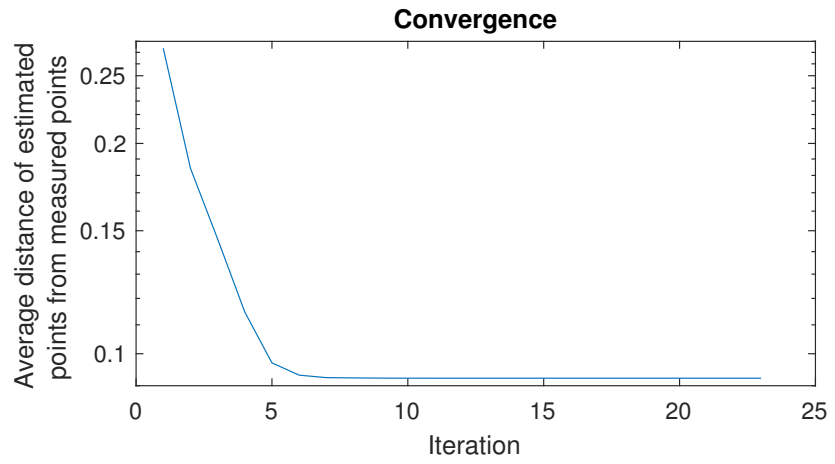

Fig. 5: Convergence speed of the method plotted on a logarithmic y-axis. An error of less than $0.1 \mathrm{~mm}$ is achieved after only 4 iterations.

2) Calibrating rotational axis: Results for the calibration of the rotation axis are similar. To show the difference between the initial estimates of the projected sphere centres 
and achieved accuracy after convergence, we show both the estimated (coloured circles) and measured (red crosses) centres in figure 6.

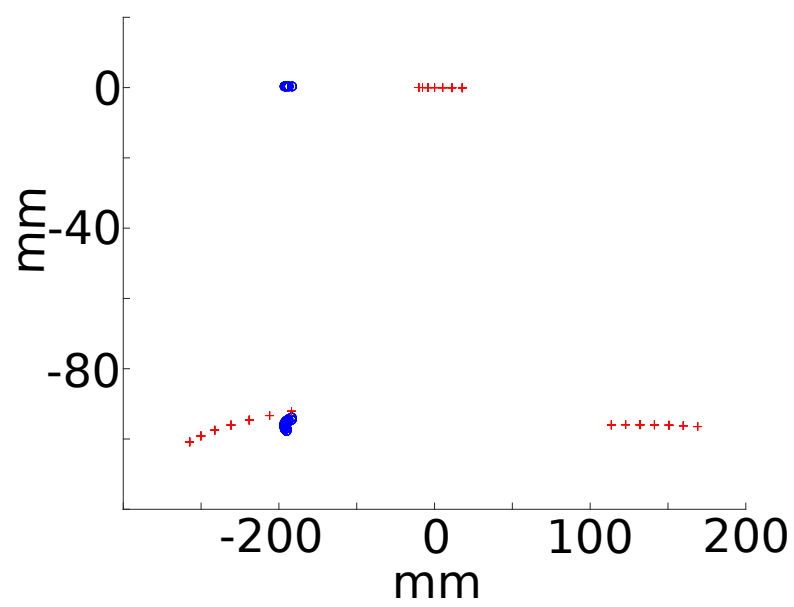

(a) After initial step

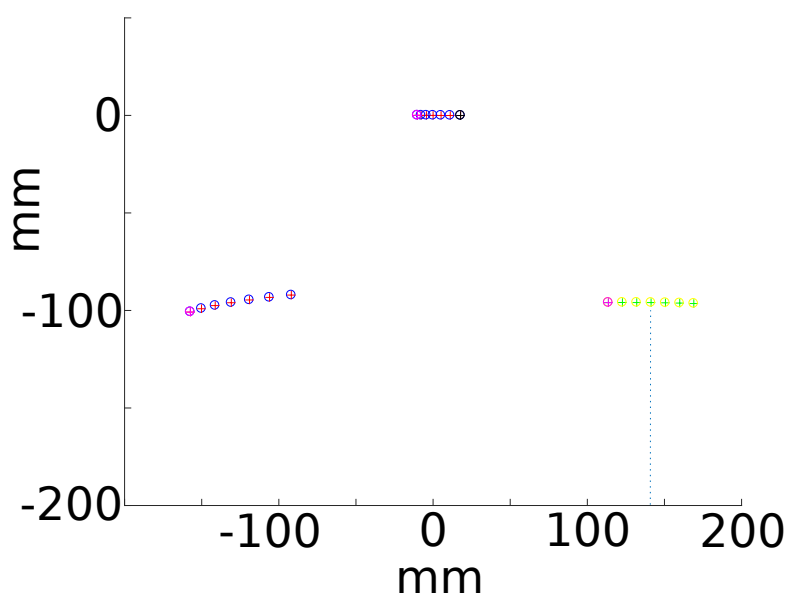

(b) After convergence

Fig. 6: Measured sphere centres on detector (red +) and estimated centres for optimised geometry (blue circle) for the three spheres used to estimate the location of the rotation axis. Average distance between observed and estimated centres is $0.033 \mathrm{~mm}$, which is a sixth of the width of a pixel.

\section{B. Performance for different scans}

The average accuracy in $\mathrm{mm}$ achieved in different scans is given in table I, which shows sub-pixel accuracy for all but one scan. Accuracy for the robot was in general worse than that for the Hexapod manipulator. Note that scan 17 was performed with a magnification of 5, whilst all other scans used a magnification of 10 . At the lower magnification, positional errors are magnified less which explains the error for scan 17 which is less than the error observed in the other two robot arm scans.

Accuracy of the limited angle calibrations, which measure the average accuracy of the sphere centres from the rotational calibration scan is significantly smaller than the errors in the raster calibration scans. This is likely to be due to the more stable rotation axis in our system. The rotation stage is mounted on top of the hexapod. Thus, when using this axis, all other axis do not move. For the raster scan, we however used the linear axis together with the hexapod to perform the required motion leading potentially to additional compound inaccuracies. We also assumed that our linear axes are linear and orthogonal and deviation form these assumptions will lead to further uncertainty in the results.

\section{Repeatability}

For some of the raster scans, we performed a calibration scan before and after the full scan. This allows us to compare repeatability of the calibration process and the repeatability of the manipulator. Table II shows the average repeatability in the estimation of the sphere centres. We used two approaches to compare repeatability. For scans 1 to 5, we performed two calibration scans, where the sphere was attached to the sample and a 3 by 3 grid of points was imaged in a single plane. We then compared the repeatability of this grid of projected points. As the spheres were removed for the full scan, the spheres were not attached to the same points for both calibration. We thus shifted the second set of points to have the same mean to the mean location of the first set of points. We then compute the difference between the two measurements and calculate a mean derivation as well as a standard deviation in this error. However, as the spheres were mounted in different locations, this introduced some additional errors due to the cone angle of the beam. For the last two scans (scans 6 and 7) we performed two full calibration scans, used these to estimate source and detector location/orientation. Once we have the two sets of estimated source and detector locations, we then used these to simulate projected points. This ensured that the same locations were used to compute the two sets of observations. Both results show that there was an error of between one and two pixels in the location of the estimated sphere centres.

\begin{tabular}{|r|r|r|r|} 
Scan & manipulator & motion & accuracy $(\mathrm{mm})$ \\
1 & hexapod & raster & 0.0923 \\
2 & hexapod & raster & 0.0981 \\
3 & hexapod & raster & 0.0424 \\
4 & hexapod & raster & 0.0627 \\
5 & hexapod & raster & 0.0628 \\
6 & hexapod & raster & 0.0639 \\
7 & hexapod & raster & 0.0537 \\
8 & hexapod & raster & 0.1187 \\
9 & robot & raster & 0.2831 \\
10 & hexapod & raster & 0.1433 \\
11 & hexapod & raster & 0.1079 \\
12 & hexapod & raster & 0.1324 \\
13 & hexapod & raster & 0.1139 \\
14 & hexapod & raster & 0.1430 \\
15 & hexapod & raster & 0.1497 \\
16 & robot & raster & 0.1736 \\
17 & robot & raster & 0.0816 \\
18 & hexapod & lim. angl. & 0.0316 \\
19 & hexapod & lim. angl. & 0.0344
\end{tabular}

TABLE I: Average accuracy after optimisation for a range of scans with different manipulators and for different scan trajectories. 
This is thus the expected spatial resolution limit for any reconstruction, as the uncertainties in the estimated source and detector locations will mean that we will have errors in the specification of our geometry of the order of a pixel.

It is interesting to observe that there do not seem to be significant differences in the errors between the robot arm and the hexapod. Also of note is the fact that the error in the repeatability seems to be large than the error in the calibration.

To investigate the influence that these errors can have on the estimation of the location of the source and detector, we conducted a simulation study where we defined a geometry similar to the geometry in the experiments used above with a magnification of about 10 . We then calculated the exact locations of a grid of points projected onto he detector. We added different amounts of gaussian noise to these points. The errors in the location of the source and the detector are shown in table III. These results are the standard derivation in the error over several repeated experiments. These errors are linear in the amount of noise added to the original locations. The error in the estimation of the source location has a standard deviation that is roughly ten times the error in the projected grid of points, whilst the error in the detector location is roughly of the same magnitude. The error in the orientation of the detector is measured not in $\mathrm{mm}$ but in radians and measures the rotation of the estimated vector relative to the true vector.

\section{Sphere centre detection performance}

We also analysed the performance of the sphere fitting procedure. We used canny edge detection and the parameters of the edge detection algorithm did not influence the performance significantly as long as we reliably estimated the edge of the sphere of interest without the inclusion of other edges, which we ensured with our masking and outlier detection approach.

The background on the projection image did however have an influence on the estimation of the sphere centre. As we attached the spheres to different samples, the variations in the

\begin{tabular}{|r|r|r|} 
Scan & manipulator & accuracy $(\mathrm{mm})$ \\
\hline 1 & robot & 0.2658 \\
2 & robot & 0.4388 \\
3 & hexapod & 0.2896 \\
4 & robot & 0.3150 \\
5 & robot & 0.1332 \\
6 & hexapod & 0.2207 \\
7 & hexapod & 0.4333
\end{tabular}

TABLE II: Error estimates in the location of the sphere centres when repeating the calibration scans.

\begin{tabular}{|r|r|r|r|r|} 
Quantity & std=0.1 & std=0.5 & std=1 & std=2 \\
\hline $\mathbf{S}$ & 1.1522 & 6.0109 & 12.5913 & 23.6311 \\
$\mathbf{D}_{0}$ & 0.1336 & 0.6985 & 1.4583 & 2.7480 \\
$\mathbf{u}$ & 0.0010 & 0.0055 & 0.0103 & 0.0182 \\
$\mathbf{v}$ & 0.0009 & 0.0048 & 0.0080 & 0.0185
\end{tabular}

TABLE III: Standard deviation of the error in the different geometric vectors for different amounts of noise added to the estimated sphere centre locations. sample's attenuation were observed to lead to edge estimates that were not always circular. Care should thus be taken when attaching the sphere to either use a part of the sample with relatively constant attenuation or to remove the sample and mount the sphere differently.

The applied Gaussian smoothing was observed to have some influence on the accuracy of the centre estimation process. To evaluate the influence of Gaussian smoothing, we looked at the error after optimisation of the geometry between the estimated sphere centres and the observed sphere centres. Results for two scans are shown in table IV for different amounts of smoothing. We see that a smoothing kernel with standard deviation of about $3 \mathrm{~mm}$ (or 15 pixels) provides the most accurate results.

\section{E. Improvement in image quality}

To compare the change in image quality with and without calibration, we did two reconstructions of a scan acquired from a carbon fibre composite panel using a raster scan trajectory. In the un-calibrated reconstruction, we assumed that the motion in the raster scan was parallel to the detector and that the detector was orthogonal to the vector from the source to the detector centre. Note that the estimation of the source to detector distance is not important as a change in this parameter simply re-scales the reconstructed volume in the source to detector distance. Results with and without calibration are shown in figure 7, where more detail is visible in the reconstruction with calibration. Image sharpness has been used previously as a measure of calibration accuracy [22]. We have measured sharpness as a fraction of high frequency image components relative to low frequency components. With this measure, we observed that calibration improved image quality for all scans we performed with calibration. Note that reconstruction of limited angle scans was not possible without an estimation of the location of the rotation axis and so for these scans, comparison to uncalibrated reconstruction was not possible.

\section{F. Conclusions}

The estimation of geometric properties for a tomographic sample manipulator system with several degrees of freedom

\begin{tabular}{|r|r|r|r|r|r|r|}
\hline Scan & & & & & & \\
\hline & std & 0.2 & 0.4 & 0.6 & 0.8 & 1 \\
\hline 1 & & 0.1207 & 0.1203 & 0.1207 & 0.1205 & 0.1187 \\
2 & & 0.0567 & 0.0557 & 0.0532 & 0.0540 & 0.0537 \\
\hline & std & 1.2 & 1.4 & 1.6 & 1.8 & 2 \\
\hline 1 & & 0.1178 & 0.1172 & 0.1138 & 0.1094 & 0.1030 \\
2 & & 0.0542 & 0.0578 & 0.0529 & 0.0569 & 0.0577 \\
\hline & std & 2.2 & 2.4 & 2.6 & 2.8 & 3 \\
\hline 1 & & 0.1022 & 0.1033 & 0.1008 & 0.1057 & 0.1038 \\
2 & & 0.0565 & 0.0559 & 0.0577 & 0.0548 & 0.0606 \\
\hline & std & 3.2 & 3.4 & 3.6 & 3.8 & 4 \\
\hline 1 & & 0.1056 & 0.1083 & 0.1143 & 0.1207 & 0.1269 \\
2 & & 0.0568 & 0.0580 & 0.0592 & 0.0598 & 0.0603 \\
\hline
\end{tabular}

TABLE IV: Average error (accuracy) after optimisation between observed and estimated sphere centres for different levels of Gaussian smoothing (std in $\mathrm{mm}$ ). 


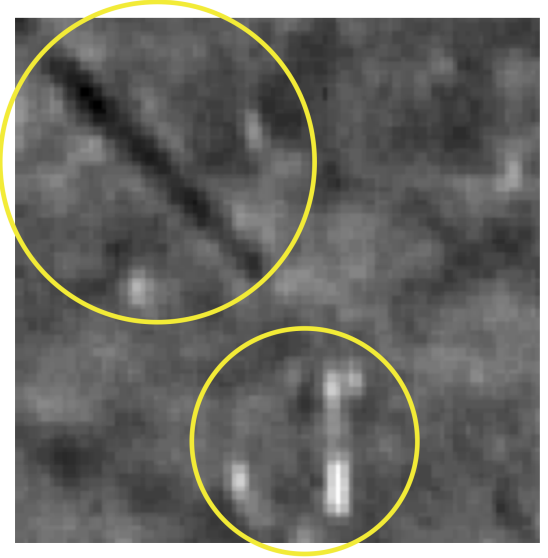

(a) Reconstruction with calibration.

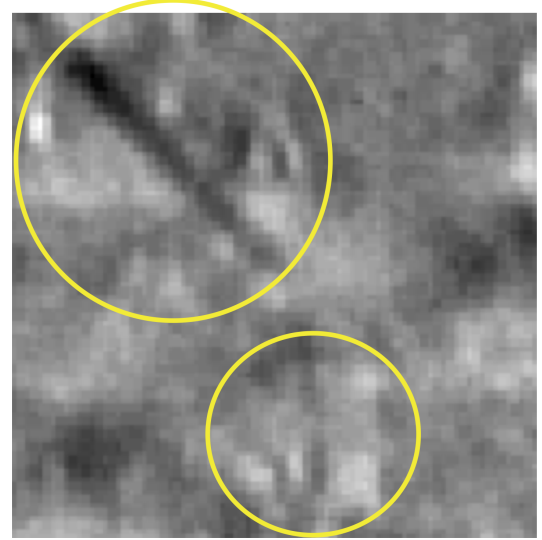

(b) Reconstruction without calibration.

Fig. 7: Detail in a slice of the reconstructed volume with (a) and without (b) calibration. The images show an approximately $2 \mathrm{~mm}$ by $2 \mathrm{~mm}$ region of a carbon fibre panel. The calibrated reconstruction more clearly shows defects of interest such as inclusions (bright spots in lower circle) and the sharper edges to air pockets (top circle).

poses several challenges. Precise geometric information is required for good volumetric reconstruction. We have explored an approach that uses one or several spheres that are imaged with the manipulator in different configurations. By choosing the configurations carefully, we could show that the projection images contain sufficient information for an accurate estimation of parameters such as source location, detector location and orientation and the alignment of linear and rotational manipulator axes. We explored this approach using two manipulator, a six axis robot arm as well as a hexapod system. Our approach allowed us to estimate geometric properties with sufficient accuracy to guarantee average geometric errors in the projected image to be below pixel size. This provided in plane spatial resolutions in our laminography scans performed with ten times magnification, that were well below 100 micrometers.

\section{REFERENCES}

[1] L. A. Feldkamp, L. C. Davis, and J. W. Kress, "Practical cone-beam algorithm," Journal of the Optical Society of America A, vol. 1, no. 6, pp. 612-619, 1984.

[2] N. O'Brian, R. Boardman, I. Sinclair, and T. Blumensath, "Recent advances in X-ray cone-beam computed laminography," Journal of $X$ ray science and technology, vol. 24, pp. 691-707, 2016.

[3] C. Wood, N. O'Brian, A. Denisov, and T. Blumensath, "Computed laminography of cfrp using an X-ray cone beam and robotic sample manipulator systems," Submitted for publication, preprint at https://eprints.soton.ac.uk/415613/, 2018.

[4] P. Grangeat, "Mathematical framework of cone beam 3d reconstruction via the first derivative of the radon transform," in Mathematical Methods in Tomography, ser. Lecture Notes in Mathematics, G. Herman, A. Louis, and F. Natterer, Eds., vol. 1497. Berlin, Germany: Springer, 1991.

[5] D. Panetta, N. Belcari, A. D. Guerra, and S. Moehrs, "An optimizationbased method for geometrical calibration in cone-beam ct without dedicated phantoms," Physics in Medicine and Biology, vol. 53, no. 14, pp. 3841-3861, 2008.

[6] Y. Kyriakou, R. Lapp, L. Hillebrand, D. Ertel, and W. Kalender, "Simultaneous misalignment correction for approximate circular conebeam computed tomography," Physics in Medicine and Biology, vol. 53, no. 22, pp. 6267-6289, 2008

[7] I. B. Tekaya, V. Kaftandjian, F. Buyens, S. Sevestre, and S. Legoupil, "Registration-based geometric calibration of industrial x-ray tomography system," IEEE Transactions on Nuclear Science, vol. 60, no. 5, 2013.

[8] F. Noo, R. Clackdoyle, C. Mennessier, T. White, and T. Roney, "Analytic method based on identification of ellipse parameters for scanner calibration in cone-beam tomography," Physics in Medicine and Biology, vol. 45, no. 11, pp. 3489-3508, 2000.

[9] C. Mennessier, R. Clackdoyle, and F. Noo, "Direct determination of geometric alignment parameters for cone-beam scanners," Physics in Medicine and Biology, vol. 54, no. 6, p. 16331660, 2010.

[10] P. Kalukin, B. Winn, Y. Wang, C. Jacobsen, Z. Levine, and J. Fu, "Calibration of high-resolution x-ray tomography with atomic force microscopy," Journal of Research of the National Institute of Standards and Technology, vol. 105, no. 6, pp. 867-874, 2000.

[11] X. Li, Z. Da, and B. Liu, "A generic geometric calibration method for tomographic imaging systems with flat-panel detectors-a detailed implementation guide," Med. Phys., vol. 37, no. 7, pp. 3844-3854, 2010.

[12] D. Scaduto and W. Zhao, "Determination of system geometrical parameters and consistency between scans for contrast-enhanced digital breast tomosynthesis," in Breast Imaging, International Workshop on Digital Mammography, 2012, pp. 24-31.

[13] H. Miau, H. Zhao, and H. Liu, "A phantom-based calibration method for digital X-ray tomosynthesis," J. X-ray Sci. Technol., vol. 20, no. 1, pp. 17-29, 2012.

[14] J. Calliste, G. Wu, P. Laganis, D. Spronk, H. Jafari, K. Olson, B. Gao, Y. Lee, O. Zhou, and J. Lu, "Second generation stationary digital breast tomosynthesis system with faster scan time and wider angular span," Med. Phys., vol. 44, no. 9, pp. 4482-4495, 2017.

[15] A. Kraemera and G. Lanza, "Assessment of the measurement procedure for dimensional metrology with x-ray computed tomography," in 14th CIRP Conference on Computer Aided Tolerancing (CAT), 2016, pp. 362367-31.

[16] J. Choi, S. Hwang, and Y. Choi, "Focal spot calibration in a digital breast tomosynthesis system," J. of the Korean Phys. Soc., vol. 60, no. 9, pp. 1457-1463, 2012.

[17] X. Li, Z. Da, and B. Liu, "Sensitivity analysis of a geometric calibration method using projection matrices for digital tomosynthesis systems." Med. Phys., vol. 38, no. 1, pp. 202-209, 2011.

[18] J. Mainprize, A. Bloomquist, X. Wang, and M. Yaffe, "Dependence of image quality on geometric factors in breast tomosynthesis." Med. Phys., vol. 38, no. 6, pp. 3090-3103, 2011.

[19] H. Banjak, M. Costin, C. Vienne, and V. Kaftandjian, "X-ray computed tomography reconstruction on nonstandard trajectories for robotized inspection," in 19th World Conference on Non-Destructive Testing, 2016.

[20] R. Clackdoyle and C. Mennessier, "Centers and centroids of the conebeam projection of a ball," Physics in Medicine and Biology, vol. 56, no. 23, pp. 7371-7391, 2011.

[21] S. Boyd and L. Vandenberghe, Convex Optimisation. Cambridge University Press, 2004.

[22] D. Panetta, N. Belcari, A. Del Guerra, A. Bartolomei, and P. Salvadori, "Analysis of image sharpness reproducibility on a novel engineered micro-ct scanner with variable geometry and embedded recalibration software." IPhys Med., vol. 28, no. 2, pp. 166-173, 2012. 